\title{
A Mechano-Chemical Model of a Solid Tumor for Therapy Outcome Predictions
}

\author{
Sven Hirsch ${ }^{1}$, Dominik Szczerba ${ }^{2,1}$, Bryn Lloyd ${ }^{1}$, Michael Bajka ${ }^{3}$, \\ Niels Kuster ${ }^{2}$, and Gábor Székely ${ }^{1}$ \\ 1 Department of Electrical Engineering, ETH, CH-8092 Zürich, Switzerland \\ ${ }^{2}$ IT'IS Foundation, CH-8004 Zürich, Switzerland \\ 3 Division of Gynecology, University Hospital Zürich, Switzerland
}

\begin{abstract}
Experimental investigations of tumors often result in data reflecting very complex underlying mechanisms. Computer models of such phenomena enable their analysis and may lead to novel and more efficient therapy strategies. We present a generalized finite element mechanochemical model of a solid tumor and assess its suitability for predicting therapy outcome. The model includes hosting tissue, tumor cells (vital and necrotic), nutrient, blood vessels and a growth inhibitor. At a certain time instant of the tumor development virtual therapies are performed and their outcomes are presented. The model is initiated with parameters either obtained directly from the available literature or estimated using multi-scale modeling. First results indicate the usefulness of multiphysics tumor models for predicting therapy response.
\end{abstract}

\section{Introduction}

According to the World Cancer Report, 12 million new cancer diagnoses are expected worldwide this year, and by 2010 it will be the leading cause of death. Better understanding tumor formation is of utmost social, economic and political importance, and finding more effective therapies may be regarded as one of the biggest challenges of our time. Computer simulation may bring new insights into the underlying mechanisms and may help to predict and optimize the effects of therapies. Simulation of such complex systems are computationally most demanding, yet the rapid development of hardware, especially emerging distributed parallel computing concepts, enable more and more realistic modeling of physiological systems. Tumor development has been studied extensively over the last three decades, reviews and exhaustive survey of the approaches can be found elsewhere 12 , or more recently in [3]. A further discussion of the relevant literature may be found in our previous works [45]. We continue to rely on the established methods from solid mechanics and chemical engineering. The finite element method (FEM) is particularly suitable for describing strain-induced cellular responses, gaining acceptance as an influential player in simulating tissue development 677. With such a representation we can account for mechanical tissue deformations induced by developing pathologies and capture its interplay with the chemical environment. We focus on benign, vascularized, solid tumor

G. Allen et al. (Eds.): ICCS 2009, Part I, LNCS 5544, pp. 715-724, 2009.

(C) Springer-Verlag Berlin Heidelberg 2009 
growth and present a significantly enhanced version of our previous model [8]. Extensions include:

1. more realistic boundary conditions for the growth factor and oxygen;

2. tumor compartmentalization;

3. deformation of the vascular system (displacement due to tumor growth);

4. effects of applying a growth inhibitor.

We do not only aim to simulate the tumor growth but also employ the model to study the effect of therapies. After describing the model we present its application to the simulation of embolization and the administration of angiostatin. Once precise experimental data are available for particular tumor types, we can integrate this quantitative knowledge into our framework and offer practical tools of clinical relevance.

\section{Definition of the Model}

The basis of our model is a set of mass and force balance equations. The mass transport of all constituents is modeled with reaction-convection-diffusion equations. Growth factor, endothelial cells, oxygen, and growth inhibitor are transported through the tissue, and may enter chemical reactions anywhere in the whole domain, which is shown schematically in Fig. 1, The components of this linked bio-chemo-mechanical model are described in the following equations where $c_{i}$ denotes a concentration of a constituent, $D_{i}$ is its diffusion coefficient and $R_{i j}$ the reaction/source term. We assume no flux boundary conditions where the inflow from the environment is not accessible.

The growth factor is produced in hypoxic regions of the tumor and decaying naturally or through inhibition:

$$
\begin{aligned}
\frac{\partial c_{1}}{\partial t} & =D_{1} \nabla^{2} c_{1}+R_{11}\left(c_{3}\right)-R_{12} c_{1}-R_{13} c_{4} \\
R_{11}\left(c_{3}\right) & = \begin{cases}R_{11}^{0}, & c_{3} \geq t_{1}^{\text {high }} \\
R_{11}^{0}+5 \cdot R_{11}^{0} \cdot\left(\frac{t_{1}^{\text {high }}-c_{3}}{t_{1}^{\text {low }}-t_{1}^{\text {high }}}\right), & t_{1}^{\text {low }}<c_{3}<t_{1}^{\text {high }} \\
6 \cdot R_{11}^{0}, & c_{3} \leq t_{1}^{\text {low }}\end{cases}
\end{aligned}
$$

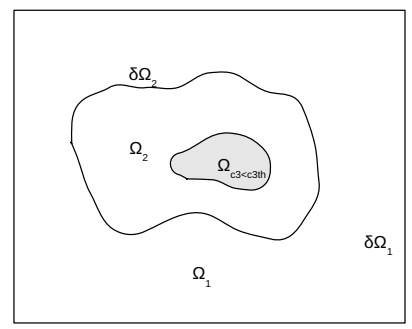

Fig. 1. Compartments of the model. The tumor $\left(\Omega_{2}\right)$ consisting of necrotic and viable part, is embedded into the host tissue $\left(\Omega_{1}\right)$. $\delta$ denotes the respective interfaces. 
with no flux boundary condition of on $\delta \Omega_{1}$. The density of endothelial cells (EC) is formulated accordingly:

$$
\begin{aligned}
\frac{\partial c_{2}}{\partial t} & =D_{2} \nabla^{2} c_{2}+\nabla \cdot\left(c_{2} \boldsymbol{u}\right)+\nabla \cdot\left(c_{2} \boldsymbol{d}\right) \\
\boldsymbol{u} & =\left(\frac{k_{0} k_{1}}{k_{1}+c_{1}} \nabla c_{1}\right)
\end{aligned}
$$

where $\boldsymbol{d}$ is a mechanical displacement field and a Dirichlet boundary condition is applied on $\delta \Omega_{1}$. The oxygen concentration in the tissue obeys a reaction-diffusion equation of the form

$$
\frac{\partial c_{3}}{\partial t}=D_{3} \nabla^{2} c_{3}+R_{31}\left(c_{3}, c_{2}\right)-R_{32}\left(c_{3}\right)
$$

with no flux boundary condition on $\delta \Omega_{1}$. Here $\left(c_{3}, c_{2}\right)$ is the source term, which depends on the vasculature and blood flow. Angiostatin (AST) is provided externally as a drug that diffuses and decays naturally:

$$
\frac{\partial c_{4}}{\partial t}=D_{4} \nabla^{2} c_{4}-R_{4} c_{4}
$$

with no flux boundary condition on $\delta \Omega_{1}$. We complete the above system of massbalance equations by adding a force-balance equation describing the mechanical stress resulting from the evolution of the system as a consequence of tumor growth (Newton equilibrium equation)

$$
\nabla \cdot \sigma+\boldsymbol{f}=0
$$

$\sigma$ is a second order tensor, $\boldsymbol{f}$ is the external force field. Here we pose a no displacement boundary condition everywhere on $\delta \Omega_{1}$ and an initial strain condition in $\Omega_{2}$.

Growth Model: Healthy tissue is in a dynamic balance between proliferation and ceasing of cells, in consequence the number of cells remains nearly constant. The process of controlled cell death - apoptosis - is an integral part of the constant renewal of tissue in the natural cell cycle. The control mechanism is part of the homeostasis required by living organisms to maintain their internal states within certain limits. The down-regulation of apoptosis in tumorous tissue leads to an over-proportional growth. Apoptosis involves a series of biochemical events. The natural mechanism of apoptosis is controlled by intrinsic and extrinsic agents. Once initiated, apoptosis results in a characteristic cell shrinkage, blebbing, and DNA fragmentation. Such, the cell may be phagocytosed safely without exposing the tissue with potentially harmful intracellular debris. In contrary, necrosis is an uncontrolled death, e.g. due to hypoxia or toxic agents, and is characterized by an uncontrolled bursting of the cell membrane with a release of the constituents. This leads to a local inflammation and only partial resorption of the debris can 
be achieved. The overall density of cells inside the tumor $N$ can be therefore decomposed into two compartments, vital $N^{+}$and necrotic $N^{-}$cells:

$$
N(t, x, y)=N^{+}(t, x, y)+N^{-}(t, x, y) .
$$

In our model, proliferation is constrained by the availability of oxygen [7.9] and space [10. We account for tumor cells proliferation, apoptosis and necrosis. Necrotic cells formed from hypoxic tumor cells. $g^{+}, g^{--}, g^{-}$are the individual growth rates for each mechanism:

$$
\begin{aligned}
& \text { growth: } g^{+}=h_{c_{3}}^{+}\left(c_{3}\right) h_{\sigma}^{+}(\sigma) \frac{\ln 2}{T_{2}^{+}}, \\
& \text {necrosis: } g^{-}=h_{c_{3}}^{-}\left(c_{3}\right) \frac{\ln 2}{T_{2}^{-}}, \\
& \text {apoptosis: } g^{--}=\frac{\ln 2}{T_{2}^{--}} .
\end{aligned}
$$

We neglected the pressure-growth dependency $h_{\sigma}^{+}=1$. Populations $N^{+}$and $N^{-}$ are

$$
\begin{aligned}
& \frac{\partial N^{+}(t, x, y)}{\partial t}=N^{+}(t, x, y)\left[g^{+}-g^{-}-g^{--}\right], \\
& \frac{\partial N^{-}(t, x, y)}{\partial t}=N^{+}(t, x, y)\left[g^{-}\right] .
\end{aligned}
$$

All the equations introduced above are solved numerically with a generalized convection-diffusion-reaction solver relying on a standard finite element discretization. The domain has been mapped with triangular meshes, typically consisting of 50-100.000 triangles.

Tissue Mechanics: Tumor progression and regression is modeled as initial strain condition. Volumetric strain is:

$$
\varepsilon_{0}(t)=\frac{d V(t)}{V(t)}=\frac{d N(t)}{N(t)} \approx \frac{\Delta N}{N(t)} .
$$

The discrete form yields the population growth $\Delta N / N$ at timestep $i$ :

$$
\frac{\Delta N}{N_{i}}=\frac{\left(N_{i+1}^{+}+N_{i+1}^{-}\right)-\left(N_{i}^{+}+N_{i}^{-}\right)}{N_{i}^{+}+N_{i}^{-}}
$$

and can also be negative, meaning cell degradation and removal. To solve the presented equations we rely on a commercial FEM package, Comsol Multiphysics. We verified this solver extensively with common benchmarks like flow past a cylinder, forced convection or a wall mounted bar sinking with gravity, where we found very good agreement with the reference solutions. 


\section{Example Application}

In this section we present an application of our model to evaluate therapeutic effects. The realism of the simulation is obviously very sensitive to the parameter choice and determination of these parameters is a challenging part of tumor simulation. The parameter set of any specific tumor type is not fully known. Some of the parameters are difficult to access by experiment, others are being reported differently by different authors. It is not the aim of this study to take precise, well-defined parameters for one particular tumor type. Instead, for this study we want to prove the feasibility of the presented modeling approach and will rely on the literature or estimates to obtain the model parameters.

\subsection{Model Parameters}

Besides the set of parameters listed in Fig. 2 we will elucidate only the ones determined via additional considerations. The delivery of oxygen depends on the partial pressure difference in the blood and the tissue. It increases in hypoxic regions, while in regions with high concentrations only little oxygen is delivered:

$$
R_{31}\left(c_{3}, c_{2}\right)=R_{0}\left(c_{2}\right)-R_{p}\left(c_{2}\right)\left(c_{3}-c_{3}^{0}\right) .
$$

The reaction term parameters $R_{0}, R_{p}$ and $c_{3}^{0}$ depend on factors such as the EC density $c_{2}$. The actual terms are derived in the next section.

$\mathrm{O}_{2}$ consumption: The actual value of oxygen consumption [1112] depends on many factors, including the tissue location, physical activity and altitude. For the consumption we assume a logistic term

$$
R_{32}\left(c_{3}\right)=R_{s a t} \frac{\left(c_{3}\right)^{p}}{\left(c_{3}\right)^{p}+\left(c_{3}^{h}\right)^{p}},
$$

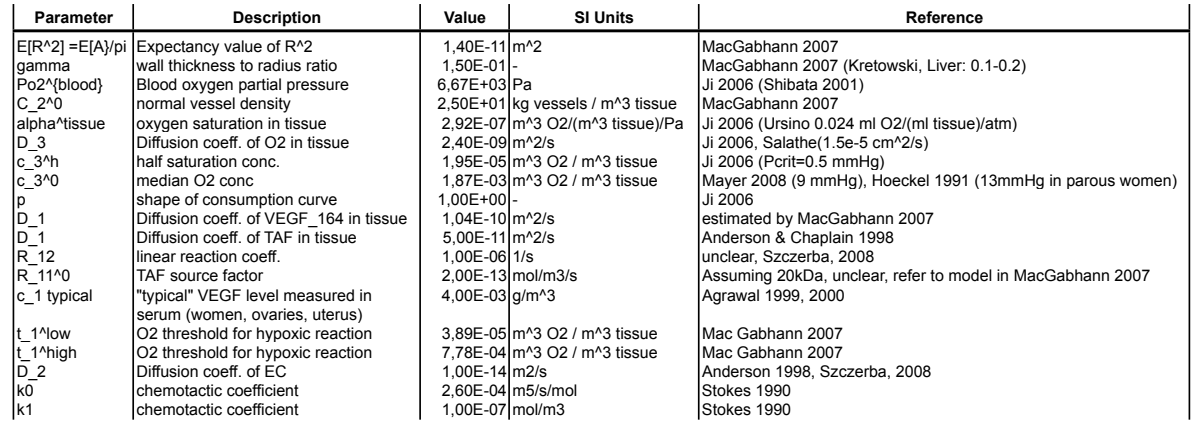

Fig. 2. Summary of the model parameters. The references not covered by the bibliography of this paper can be found in an earlier publication [8]. 
where $c_{3}^{h}$ is the concentration at which the reaction term reaches half maximum and $p$ controls the shape of $R_{32}\left(c_{3}\right)$. This reflects the fact that the consumption is bounded by the amount of oxygen available but also saturates if oxygen is unlimited.

Oxygen Source Terms: The approximate form of the oxygen source terms can be estimated based on following observations. For a vessel segment of length $L$, radius $R_{s}$ and wall thickness $w_{s}$ the exchange of oxygen with the surrounding tissue depends on the oxygen gradient, respectively the partial pressure difference across the vessel wall (Fick's first law)

$$
Q_{\mathrm{O}_{2}}=d S D_{\mathrm{O}_{2}}^{w} \alpha_{\mathrm{O}_{2}}^{w}\left(P_{\mathrm{O}_{2}}^{\text {blood }}-P_{\mathrm{O}_{2}}^{\text {tissue }}\right) \frac{1}{w_{s}},
$$

where $d S=2 \pi R_{s} L$ is the surface of the vessel segment and $D_{\mathrm{O}_{2}}^{w}$ is the diffusion constant in the vessel wall. Oxygen concentration in the tissue is proportional to the oxygen partial pressure according to Henri's law

$$
P_{\mathrm{O}_{2}}^{\text {tissue }}=c_{3} / \alpha_{\mathrm{O}_{2}}^{\text {tissue }}
$$

where the parameter $\alpha_{\mathrm{O}_{2}}^{\text {tissue }}$ is the oxygen solubility. The term $\alpha_{\mathrm{O}_{2}}^{w}\left(P_{\mathrm{O}_{2}}^{\text {blood }}-\right.$ $\left.P_{O_{2}}^{\text {tissue }}\right) \frac{1}{w_{s}}$ is the oxygen concentration gradient across the vessel wall. For a short segment, the partial pressure in tissue $P_{\mathrm{O}_{2}}^{\text {tissue }}$ and the blood $P_{\mathrm{O}_{2}}^{\text {blood }}$ is approximately constant. In a given volume $V$ the total oxygen delivered to the tissue is the sum of contributions from all segments within $V$

$$
Q_{O_{2}}^{V}=2 \pi L \sum_{k}^{N} R_{k} D_{O_{2}}^{w} \alpha_{O_{2}}^{w}\left(P_{O_{2}}^{\text {blood }}-P_{O_{2}}^{\text {tissue }}\right) \frac{1}{w_{k}} .
$$

The EC density $c_{2}$ includes the vessel wall and lumen, within the volume $V$ it is

$$
c_{2}=\frac{1}{V} \sum_{k}^{N} L \cdot R_{k}^{2} \pi=\frac{L}{V} \sum_{k} R_{k}^{2} \pi \text {. }
$$

Solving (21) for the segment length $L$ and inserting the result in (20), gives a relation between the oxygen flow $Q_{\mathrm{O}_{2}}^{V}$ and EC density. Under the assumptions of constant blood oxygen partial pressure and constant wall thickness to radius ratio $\gamma$, the terms can be simplified further. Finally, the source terms can be deduced by dividing the oxygen flow by the volume $V$

$$
R_{31}\left(c_{3}, c_{2}\right)=\frac{Q_{\mathrm{O}_{2}}^{V}}{V}=c_{2} \frac{2 / \gamma \cdot D_{\mathrm{O}_{2}}^{w} \alpha_{\mathrm{O}_{2}}^{w}\left(P_{O_{2}}^{\text {blood }}-P_{\mathrm{O}_{2}}^{\text {tissue }}\right)}{\sum_{k} R_{k}^{2} / N}
$$


We recognize the term $\sum_{k} R_{k}^{2} / N$ as the expected value $E\left[R^{2}\right]$.

$$
\begin{aligned}
R_{31}\left(c_{3}, c_{2}\right) & =c_{2} \frac{2 / \gamma \cdot D_{O_{2}}^{w} \alpha_{O_{2}}^{w} P_{O_{2}}^{\text {blood }}}{E\left[R^{2}\right]}-c_{2} \frac{2 / \gamma \cdot D_{O_{2}}^{w} \alpha_{O_{2}}^{w} / \alpha_{O_{2}}^{\text {tissue }} \cdot c_{3}}{E\left[R^{2}\right]} \\
& =R_{A} c_{2}-R_{B} c_{2} c_{3}
\end{aligned}
$$

It is easy to verify that the derived source term corresponds to the generic form proposed in (16) with appropriate selection of the terms $R_{0}$ and $R_{p}$.

\subsection{Initialization and Growth}

The simulations are initiated with a small cluster of tumor cells in the center of the host tissue surrounded by vessels on each side. The corresponding high EC density is visible in Fig. 4 as a decaying gray shadow gradient at the box walls. The tumor promotes directed vessel growth via secretion of tumor angiogenic factors (TAF), leading to EC migration from the adjacent parent vessels. In this vascular phase the tumor expands virtually unbounded and will eventually cause physiological problems due to compression of the surrounding tissue. The EC density increase the corresponding vessels' diameter and wall thickness in time, which in turn modulates their oxygen delivery rate. For large vessels, blood flow increases and diffusion through the wall decreases. For capillaries it is the contrary: most of oxygen exchange is realized here, but the net mass flux is small. To obtain the distributions of vessel diameters we solve a Dirichlet problem inside the domain by fixing the vessel diameters at the boundaries to the expected diameters (feeding arterioles, $50 \mu \mathrm{m}$ ). The equation parameters are determined experimentally to achieve capillaries of about $5 \mu \mathrm{m}$ radius in the center of the domain. The unregulated tumor growth is presented in Fig.4left (EC) and Fig. 3 (oxygenation map). Non-symmetrical compartments develop despite the initial boundary symmetry. EC density is realized with a typical capillary buildup in the center of the host tissue in form of a frequently observed vascular capsule.
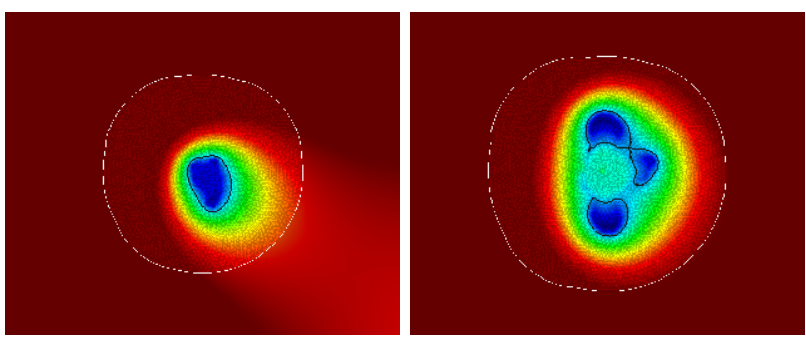

Fig. 3. Examples of compartment formation within the tumor (white outline with mesh) of necrotic and viable tissue for different necrosis thresholds. The oxygenation map is color coded, the inner areas denote oxygen isocontours of the threshold level. 


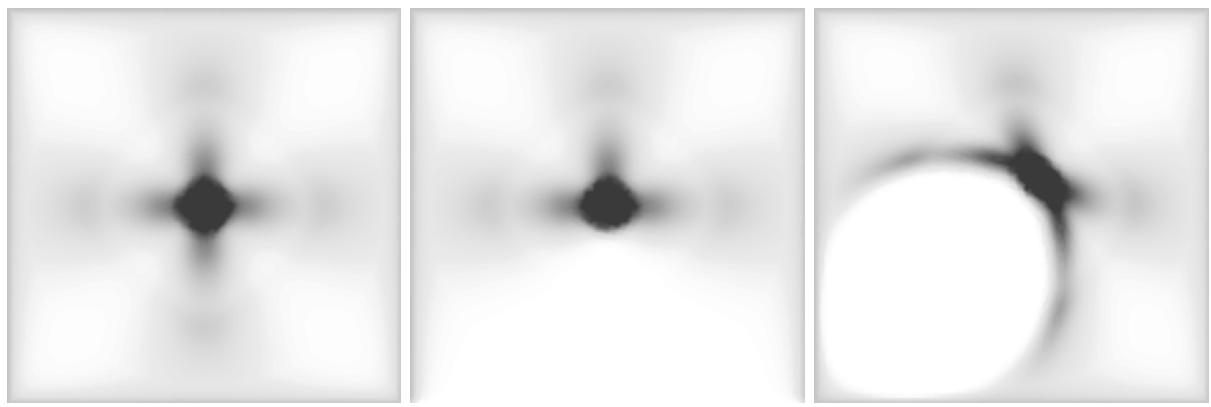

Fig. 4. EC density within the host tissue. Left: unregulated growth; center: embolization of the lower vessel; right: tumor after angiostatin infusion.

Four branches of vessels are clearly visible, connecting the tumor to the feeding arteries on the periphery. The tumor itself is penetrated by a dense network of capillaries, as often actually observed.

Growth inhibition through embolization: Embolization is a minimally-invasive procedure involving a selective occlusion of blood vessels, by introducing an artificial embolus (coil, particle, foam, plug). The purpose of embolization is to permanently block the blood flow to the tumor, leading to immediate tissue hypoxia and eventually necrosis. In our simulation the tumor grows initially unbounded, supported by the accompanying angiogenesis for 4 months. The virtual embolization procedure is performed by disabling the lower feeding artery. We implement it by replacing the Dirichlet boundary condition by a non-penetration condition. Note, that we do not remove any existing daughter vessels that sprouted off. This relatively well approximates the fact that only blood supply is eliminated, but neither the blood vessels nor endothelial cells. The EC density concentration 6 months after treatment in Fig. 4 (center) shows an asymmetric appearance. The vessel density in the lower half is greatly reduced, which corresponds to a ceasing

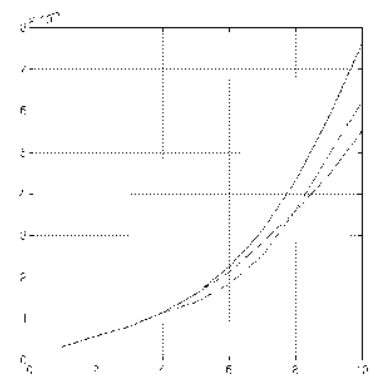

Fig. 5. Development of total tumor mass under different conditions. Solid line: no treatment; dashed line: Angiostatin treatment; dot dashed line: embolization. 
of the respective feeding vessel. The capillary core is still maintained, and shows only slight signs of asymmetry in the lower part. Obviously the capillaries still sufficiently supply the tumor with oxygen, such that the vessel network can be maintained through preexisting anastomosis. As the daughter vessels were not removed, they are still a part of a network and continue to deliver oxygen. In Fig. [5 we can, however, observe that the total mass of the tumor is somewhat lower than in the unregulated case.

Growth inhibition through Angiostatin: Next we test the infusion of angiostatin (AST), a potent inhibitor of angiogenesis. There are several ways angiostatin influences the tumor and the underlying mechanisms are not fully understood 1314. In the study we introduce angiostatin locally in the 4 th month of tumor development and continue its administration till the end of month 10 . This inhibitor neutralizes the growth factor directly as described by (2). We apply AST off-center in the lower left quadrant and not inside the tumor, to prevent the vessels from reaching the tumor at all. In Fig. 4 (right) we notice a strongly asymmetric EC density distribution, where the AST supplied area is excluded from any vessel growth. The local concentration of AST does not actually prevent the vessels from connecting to the tumor. Instead, the EC density builds clearly defined feeding vessels around the AST supplied area. The EC density accumulates outside the AST application region and is able to form a dense capillary capsule. We were not able to find data in the literature for the reaction rate between AST and the TGF, and it was arbitrarily set 1:1, justified to some extent by the similar molecular weights of the two factors.

\section{Discussion and Outlook}

The results from our extended model are in a reasonable qualitative agreement with commonly available findings. The improved modeling of EC added further physiological realism, since we managed to model the capillary penetration of the tumor and are now able to achieve tumor compartmentalization as seen in Fig. 3 . This approach generates reasonable results, which make it a viable alternative to the expensive explicit modeling of the vessels. The origin of asymmetric phenotypes from symmetric boundary conditions we will consider closely with refined meshes. We will further extend the scope of our model, to cover also other aspects like thermal effects, hormonal, radiation or chemotherapy. The tumor model is generally difficult to validate since many of the parameters are unknown or carry large measurement errors. It is the most difficult challenge to quantify these effects on the cellular level, which is the reason why the simulation outcome can not be currently validated against the physiological findings. After all the development of the tumor may be assessed on the macroscopic level, comparing the tumor regression to in vivo diagnostic observation (CT, US, MRI). We are convinced of the great value of the computational model once parameters are available from measurements. Here we are especially interested in verifying mechanical effects as it is one of the major benefits of relying on FEM methods for the simulation. 
Acknowledgments. This work is part of the Swiss National Center of Competence in Research on Computer Aided and Image Guided Medical Interventions (NCCR Co-Me), supported by the Swiss National Science Foundation.

\section{References}

1. Araujo, R.P., McElwain, D.L.S.: A history of the study of solid tumour growth: the contribution of mathematical modelling. Bulletin of Mathematical Biology 66(5), 1039-1091 (2004)

2. Byrne, H.M., Alarcon, T., Owen, M.R., Webb, S.D., Maini, P.K.: Modelling aspects of cancer dynamics: a review. Philos. Transact. A Math. Phys. Eng. Sci. 364(1843), 1563-1578 (2006)

3. Bellomo, N., Li, N., Maini, P.: On the foundation of cancer modelling: Selected topics, speculations, and perspectives. Mathematical Models and Methods in Applied Sciences 18(4) (2008)

4. Szczerba, D., Székely, G., Kurz, H.: A multiphysics model of capillary growth and remodeling. Simulation of Multiphysics Multiscale Systems (2006)

5. Lloyd, B.A., Szczerba, D., Rudin, M., Székely, G.: A computational framework for modelling solid tumour growth. Philos. Transact A. Math. Phys. Eng. Sci. (July 2008)

6. Gordon, V.D., Valentine, M.T., Gardel, M.L., Andor-Ardo, D., Dennison, S., Bogdanov, A.A., Weitz, D.A., Deisboeck, T.S.: Measuring the mechanical stress induced by an expanding multicellular tumor system: a case study. Experimental Cell Research 289(1), 58-66 (2003)

7. Graziano, L., Preziosi, L.: Mechanics in tumor growth. In: Mollica, F., Rajagopal, K., Preziosi, L. (eds.) Modelling of Biological Materials, pp. 267-328. Birkhäuser, Basel (2007)

8. Szczerba, D.: A multiphysics model of myoma growth. In: Bubak, M., van Albada, G.D., Dongarra, J., Sloot, P.M.A. (eds.) ICCS 2008, Part II. LNCS, vol. 5102, pp. 187-196. Springer, Heidelberg (2008)

9. Anderson, A.R., Chaplain, M.A.: Continuous and discrete mathematical models of tumor-induced angiogenesis. Bulletin of Mathematical Biology 60(5), 857-899 (1998)

10. Chaplain, M.A.J., Graziano, L., Preziosi, L.: Mathematical modelling of the loss of tissue compression responsiveness and its role in solid tumour development. Math. Med. Biol. 23(3), 197-229 (2006)

11. Salathe, E.P., Xu, Y.H.: Non-linear phenomena in oxygen transport to tissue. Journal of Mathematical Biology 30(2), 151-160 (1991)

12. Ji, J.W., Tsoukias, N.M., Goldman, D., Popel, A.S.: A computational model of oxygen transport in skeletal muscle for sprouting and splitting modes of angiogenesis. J. Theor. Biol. 241(1), 94-108 (2006)

13. Sim, B.K., MacDonald, N.J., Gubish, E.R.: Angiostatin and endostatin: endogenous inhibitors of tumor growth. Cancer Metastasis Rev. 19(1-2), 181-190 (2000)

14. Folkman, J.: Role of angiogenesis in tumor growth and metastasis. Semin. Oncol. 29(6 Suppl 16), 15-18 (2002) 